\title{
Elaboração e avaliação de um instrumento para identificar memórias referentes à Unidade de Terapia Intensiva
}

\author{
Development and evaluation of an instrument to \\ identify memories on the Intensive Care Unit \\ Jaquilene Barreto da Costa', Sonia Silva Marcon²
}

\section{RESUMO}

Objetivo: Descrever o desenvolvimento de um instrumento para identificar as recordações de pacientes internados em Unidade de Terapia Intensiva (UTI) e verificar a validade de conteúdo e a aplicabilidade do instrumento. Método: Estudo de desenvolvimento metodológico, fundamentado na teoria de elaboração de escalas psicológicas de Pasquali (1998). Para elaboração do instrumento, foram percorridas três etapas, a construção dos itens, a análise da validade de conteúdo e aparência por um painel de experts e, por fim, a realização de um estudo-piloto para verificar a aplicabilidade do instrumento na população-alvo. Resultados: Houve pouca discordância entre os experts na análise das questões de acordo com os domínios do construto. Os itens foram avaliados positivamente, recebendo uma pontuação acima de $80 \%$, valor este que obedece ao critério de validação do instrumento. Dessa forma, os itens foram considerados consistentes em relação aos domínios e ao construto, possibilitando identificar recordações das experiências vividas pelos pacientes durante sua permanência em UTI. Conclusões: Os itens propos-

\section{Palavras-chave}

Estudos de validação, Unidades de Terapia Intensiva, memória, ilusões. tos mostraram-se compreensíveis, claros e com uma adequada estrutura de apresentação. O instrumento apresenta validade de conteúdo atestada por um painel de experts, demonstrando possuir características teóricas para identificar memórias de pacientes internados em UTI a curto e longo prazo.

\section{ABSTRACT}

Objective: Describe the development of an instrument to identify records of their stay at the intensive care unit, and verify content validation and the applicability of the instrument. Method: It is a methodological study, based in Pasquali's (1998) psychological scale elaboration theory. To elaborate the instrument, three stages were followed: the built of items; appearance and content validation analysis performed by a panel of experts; together with implementation of a pilot study to validation instrument applicability in the targeted population. Results: There was

1 Universidade Estadual de Maringá (UEM), Programa de Pós-graduação em Ciências da Saúde. 2 Universidade Estadual de Maringá (UEM), Núcleo de Estudo, Pesquisa, Assistência e Apoio à Família (NEPAAF).

Recebido em

3/9/2009

Aprovado em

$10 / 11 / 2009$
Endereço para correspondência: Jaquilene Barreto da Costa Hospital Universitário do Oeste do Paraná-HUOP - Setor de Psicologia Av. Tancredo Neves, 3224, Santo Onofre - 85804-260 - Cascavel, PR E-mail: jaquihuop@yahoo.com.br 


\section{Keywords}

Validation studies, Intensive Care Units, memory, illusions. little unconformity among the experts when analyzing the questions according to the domain of the constructor. The items were positively evaluated having a punctuation value above $80 \%$, which abides the instrument validation criteria. Thus, the items were considered consistent in relation to the domain and constructor allowing identification of recollection of memories lived by the patients during their stay at the ICU. Conclusions: The proposed items demonstrated to be comprehensive, clear, and having an adequate presentation structure. The instrument shows validation to the content certified by a panel of experts, thus demonstrating to have theoretical characteristics to identify short and long term recollections of ICU patients.

\section{INTRODUÇÃO}

As experiências e as recordações relatadas por pacientes internados em Unidade de Terapia Intensiva (UTI), especialmente aqueles que se submeteram à ventilação artificial e à sedação, têm constituído foco de vários estudos ${ }^{1-3}$. Pacientes avaliados após a alta dessa unidade relatam experiências de fatos reais e também apresentam memórias ilusórias ${ }^{4-5}$.

Alguns pacientes apresentam recordações vívidas de suas experiências na UTI. Experiências negativas incluem recordações de dor, ansiedade, dificuldade de sono, alucinações e alterações no funcionamento cognitivo ${ }^{6}$.

Por outro lado, estudos têm demonstrado que pacientes internados em UTI têm recordações limitadas de eventos reais referentes à sua permanência naquela unidade e muitos são incapazes de descrever suas recordações e aproximadamente $5 \%$ deles têm pouca ou nenhuma memória, mas podem relembrar uma variedade de sonhos, alucinações e pesadelos, que são com frequência de natureza persecutória ${ }^{7-8}$. A maioria dos pacientes (55\%) tem uma combinação de memórias de eventos reais, alucinações ou pesadelos 5 .

Vivências de sonhos, alucinações e pesadelos são fontes de desconforto, estresse e ansiedade para pacientes após a alta da UTI'. Por essa razão, as recordações apresentadas pelos pacientes após a alta dessa unidade poderão ter um impacto na sua recuperação e alguns pacientes podem continuar afetados por vários meses ${ }^{10-11}$. Por outro lado, pacientes sem recordações de fatos reais de sua permanência na UTI e apenas com memórias ilusórias (alucinações, sonhos, pesadelos) apresentam alterações emocionais significativas após a alta hospitalar'2.

As pesquisas sobre memórias de pacientes de UTI têm revelado resultados inconsistentes ${ }^{2,6-8}$, tanto em relação à capacidade do paciente em recordar as experiências da internação quanto ao conteúdo das recordações apresentadas. Isso pode ser explicado devido ao fato de esse evento ter sido estudado em períodos distintos após a alta, com diferentes instrumentos, metodologias e coortes de pacientes.

A criação de um instrumento implica rigor metodológico, etapas bem definidas e procedimentos precisos. Pasquali16 descreve minuciosamente os passos e procedimen- tos metodológicos para construção de escalas psicológicas. A proposta metodológica para construção e validação do instrumento ora proposto foi realizada a partir da adoção de alguns critérios desse modelo, com ajustes de alguns pontos do processo de validação, tendo em vista o objetivo do estudo.

Atualmente, não há instrumentos nacionais padronizados para avaliar as experiências e os resultados das recordações referentes à UTI em curto e longo prazo. Além disso, estudos têm demonstrado ${ }^{9,12}$ que pacientes que não possuem recordações de fatos reais de sua permanência na UTI, mas apenas memórias ilusórias, como alucinações, sonhos e pesadelos, apresentaram altas taxas de sintomas do transtorno de estresse pós-traumático, ansiedade e depressão, após a alta hospitalar. Assim, considerando o impacto desse evento para a saúde mental do paciente, a avaliação e a identificação precoce das memórias referentes à UTI têm se configurado como relevantes e necessárias, tendo em vista a prevenção de subsequentes morbidades psiquiátricas.

Diante disso, definiu-se como objetivo do estudo desenvolver um instrumento para identificar as recordações de pacientes após a alta da UTI, fazer a validação de conteúdo e testar a aplicabilidade do instrumento.

\section{METODOLOGIA}

Estudo de desenvolvimento metodológico, de abordagem quantitativa, realizado no período de agosto de 2008 a janeiro de 2009.

\section{Elaboração do instrumento}

A elaboração do instrumento de medida foi fundamentada na teoria de elaboração de escalas psicológicas de Pasqua$\mathrm{li}^{16}$, com base em três conjuntos de procedimentos: teóricos, empíricos e analíticos. O primeiro pólo enfoca a elaboração do construto ou objeto a ser estudado para o qual se quer desenvolver um instrumento de medida, além da operacionalização do objeto de estudo em itens. O segundo define as etapas e as técnicas da aplicação do instrumento-piloto e, por fim, o procedimento analítico estabelece a análise esta- 
tística a ser realizada após a coleta dos dados, tendo em vista a validação do instrumento.

Para a elaboração do construto, consideraram-se estudos anteriores com pacientes de UTI que tinham como objetivo avaliar recordações e experiências de sua internação ${ }^{8,13-14}$, além de consulta com peritos da área e da própria experiência da pesquisadora.

A escolha das questões do instrumento proposto foi baseada no conteúdo de alguns itens apresentados na versão inglesa do instrumento Intensive Care Unit Memory - ICUM, que avalia as recordações de pacientes antes e após a internação na UTI, consiste de 14 itens, dos quais dois são para determinar sintomas do transtorno de estresse pós-traumático ${ }^{15}$. No estudo, para validação desse instrumento, foi avaliada uma coorte prospectiva de 45 pacientes de uma UTI geral e os testes iniciais de validade e confiabilidade do instrumento se mostraram favoráveis, uma vez que foi encontrada boa confiabilidade teste-reteste relativa à memória de fatos reais e memória de ilusão (sonhos, pesadelos, alucinações). No entanto, memórias de sentimentos não foram consistentes ao longo dos quatro meses.

Para elaboração do instrumento, foram percorridas três etapas, a construção dos itens, a análise da validade de conteúdo e aparência dos itens por um painel de experts e, por fim, a realização de um estudo-piloto para verificar a aplicabilidade do instrumento na população-alvo.

O instrumento ora proposto é do tipo checklist e consiste de itens que investigam as recordações dos pacientes durante sua internação na UTI. Dois itens do instrumento (itens 2 e 9) são perguntas abertas propostas para possibilitar o resgate das experiências vividas pelo paciente. Recordação ou memória nesse estudo foi definida como a capacidade do paciente de lembrar-se de qualquer evento relacionado ao tratamento, ambiente ou experiência emocional em que ele acreditou ter ocorrido durante o período de recuperação da consciência.

O conteúdo semântico dos itens abrange quatro domínios principais: as recordações relacionadas ao tratamento, ao ambiente físico (quando o paciente tem recordações das visitas dos familiares, do ruído, do tubo, dos procedimentos etc.); aspectos relacionados a memórias de experiências emocionais (sentir-se ansioso, com medo, confuso, dor etc.); e aquelas relacionadas a memórias irreais ou de ilusão (sonhos, pesadelos, alucinação, delírios). Os tipos de memórias foram classificados como amnésia total - aqueles pacientes que não têm nenhuma recordação do período de internação; memórias de fatos reais - aquelas recordações relacionadas a fatos reais referentes ao tratamento, ambiente, aspectos emocionais e memórias de ilusão, relacionadas a eventos ilusórios, como pesadelos, sonhos e alucinações.

\section{Validação do instrumento}

A validade de conteúdo é um método baseado no julgamento. Para isso, foi selecionado um grupo de juízes, com expe- riência em UTI, cuja tarefa foi analisar os itens e julgar se eles eram abrangentes e representativos e, ainda, se o conteúdo de cada item se relacionava com o construto em questão.

Para determinar a validade de conteúdo do instrumento, os itens propostos foram submetidos à análise de juízes (experts na área do construto) e à análise semântica que objetivou verificar se os itens eram compreensíveis e se apresentavam validade aparente ou de face que consiste no julgamento dos itens quanto à clareza, à facilidade de leitura, à compreensão e à forma de apresentação do instrumento.

\section{Composição do painel de experts}

Para composição do painel de experts, foi realizado um levantamento de pesquisadores com experiência em UTI na Plataforma Lattes, no site do Conselho Nacional de Desenvolvimento Científico e Tecnológico (CNPq). Foram selecionados intencionalmente 17 profissionais de várias regiões do Brasil, considerando o tempo de atuação na referida unidade e o fato de eles constituírem referência neste campo de atuação e, portanto, conhecedores das questões estudadas.

Os 17 experts localizados foram convidados a participar da validação do instrumento por meio de correspondência encaminhada via e-mail. Eles eram profissionais de diversas áreas, sendo três médicos, quatro enfermeiras e quatro psicólogos, todos com mais de cinco anos de atuação em Unidade de Terapia Intensiva. Destes, seis atuavam tanto na assistência ao paciente quanto no ensino e na pesquisa em terapia intensiva e sete trabalhavam em instituição pública.

Aos onze que aceitaram participar do estudo foi encaminhado o instrumento de pesquisa, juntamente com um guia contendo instruções de como proceder para respondê-lo, conforme proposto por Pasquali16, sendo solicitado a estes que, após a avaliação do instrumento, encaminhasse seu parecer e sugestões via e-mail. Foi dado um prazo de 20 dias a partir do envio da carta e aqueles que não responderam ao convite não foram mais contatados. O contato com os experts e a avaliação do instrumento se deram nos meses de agosto a setembro de 2008.

$\mathrm{Na}$ análise do construto, os experts julgaram o quanto cada característica do construto foi coberta pelos itens propostos. Para isso, cada item do instrumento foi avaliado de acordo com os quesitos: objetividade, simplicidade, relevância, precisão, credibilidade, clareza, adequação da linguagem e grau de dificuldade, utilizando, para tanto, uma escala do tipo likert de 5 pontos, sendo 1 muito ruim e 5 muito bom. Uma concordância de, pelo menos, $80 \%$ entre os juízes serviu de critério de decisão para determinar a qualidade do item.

\section{Análise dos itens}

De acordo com a pontuação preestabelecida para avaliação dos itens do instrumento pelos experts, sendo 1 muito ruim, 2 ruim, 3 razoável, 4 bom e 5 muito bom, realizou-se uma conversão dos escores 1 e 2 para o escore 1 e dos escores 4 e 5 
para o escore 5. O escore 3 permaneceu inalterado. Assim, a pontuação usada para análise dos dados ficou constituída dos escores 1 (muito ruim/ruim), 3 (razoável) e 5 (bom/muito bom).

Após a avaliação dos experts e ajustamento de alguns itens, completaram-se os procedimentos teóricos referentes à construção do instrumento de medida. Desse processo resultou o instrumento-piloto, o qual se constituiu da hipótese a ser empiricamente testada. A tarefa de testagem foi iniciada com os procedimentos experimentais, os quais consistiram em coletar informações empíricas válidas e submetê-las às análises estatísticas pertinentes.

\section{Teste-piloto}

Com base no que foi apresentado, a próxima etapa de validação foi testada por meio da aplicação do instrumento, tendo em vista sua aplicabilidade e validade semântica (inteligibilidade e compreensão) dos itens pela população-alvo. Essa aplicação foi realizada com pacientes atendidos no período de outubro a dezembro de 2008 no Ambulatório de Seguimento pós-UTI de um Hospital Escola localizado na Região Oeste do Paraná. A escolha do referido local se deu porque este é o único ambulatório de acompanhamento pós-UTI da região. O retorno de pacientes no ambulatório ocorre três meses após a alta da UTI e a avaliação é feita por equipe multidisciplinar constituída por médico, psicólogo, fisioterapeuta, nutricionista, fonoaudiólogo e assistente social. A aplicação do instrumento foi realizada pela pesquisadora e por uma psicóloga devidamente treinada que atende no referido ambulatório.

Na avaliação das recordações, foram consideradas ausência total de memória (amnésia total), memórias de fatos reais (recordações do tratamento/ambiente/emocional) e memórias ilusórias (pesadelos, alucinações, sonhos). Se um ou mais itens foram recordados pelo paciente, este foi definido como apresentando memória da UTI.
Após o envio do material pelos experts e a realização do estudo-piloto, as respostas foram digitadas em um banco de dados do programa Microsoft ${ }^{\oplus}$ Excel $^{\circledast} 2002$ e analisadas por meio de frequência simples.

O estudo ora apresentado é parte do projeto de dissertação de mestrado e ocorreu em conformidade com as diretrizes estabelecidas na Resolução 196/96 do Conselho Nacional de Saúde, o qual foi submetido à apreciação do Comitê de Ética da Universidade Estadual de Maringá (UEM) e aprovado mediante o Parecer no. 461/2008. Todos os participantes assinaram o Termo de Consentimento Livre e Esclarecido.

\section{RESULTADOS}

\section{Validade de conteúdo}

A pontuação atribuída pelos juízes permitiu identificar a adequação do instrumento quanto à validade de conteúdo e à compreensão dos itens, conforme apresentado na tabela 1.

Observa-se que houve pouca discordância entre os juízes na análise das questões de acordo com os domínios do construto. A maioria dos itens foi avaliada positivamente, recebendo uma pontuação acima de $80 \%$, valor este que obedece ao critério de validação desse instrumento. Dessa forma, os itens foram considerados consistentes em relação aos domínios definidos e em relação ao construto.

Cabe salientar, no entanto, que no item seis os critérios objetividade, simplicidade, credibilidade, clareza e adequação da linguagem foram avaliados com uma concordância de apenas $72,7 \%$ entre os juízes, e dois juízes avaliaram esse item na maioria dos critérios como sendo ruim ou razoável. Dessa forma, em relação a esse item, foram acatadas as sugestões de mudança em relação à forma de apresentação e ao uso de linguagem mais adequada.

Tabela 1. Percentual de concordância entre juízes em relação à análise das questões do instrumento para identificar memórias de UTI. Cascavel (PR), 2008

\begin{tabular}{|c|c|c|c|c|c|c|c|c|}
\hline \multirow{3}{*}{ DOMÍNIOS } & \multicolumn{8}{|c|}{ CRITÉRIOSDE ANÁLISE DE CONTEÚDO } \\
\hline & \multirow[t]{2}{*}{ Itens } & Objetividade & Simplicidade & Relevância & Precisão & Credibilidade & Clareza & $\begin{array}{c}\text { Adequação da } \\
\text { linguagem }\end{array}$ \\
\hline & & $\%$ & $\%$ & $\%$ & $\%$ & $\%$ & $\%$ & $\%$ \\
\hline \multirow{2}{*}{ Memórias gerais da UTI } & 1 & 100 & 100 & 100 & 100 & 100 & 100 & 100 \\
\hline & 2 & 81,8 & 100 & 100 & 100 & 90,9 & 90,9 & 100 \\
\hline Memórias do tratamento & 3 & 100 & 90,9 & 100 & 100 & 100 & 100 & 90,9 \\
\hline Memórias do ambiente & 4 & 100 & 100 & 100 & 100 & 100 & 100 & 100 \\
\hline Memórias emocionais & 5 & 100 & 100 & 100 & 100 & 100 & 100 & 100 \\
\hline \multirow{2}{*}{ Memórias de ilusão } & 6 & 72,7 & 72,7 & 90,9 & 81,8 & 72,7 & 72,7 & 72,7 \\
\hline & 7 & 100 & 100 & 100 & 100 & 100 & 100 & 100 \\
\hline Memórias do estado mental & 8 & 100 & 100 & 100 & 72,7 & 90,9 & 100 & 100 \\
\hline Memórias de experiências desagradáveis & 9 & 90,9 & 90,9 & 100 & 90,9 & 100 & 100 & 100 \\
\hline
\end{tabular}


Quanto ao critério relevância e precisão, nenhum dos itens recebeu um escore abaixo de três, considerado este como ruim e muito ruim. $\mathrm{O}$ item oito obteve no quesito precisão porcentagem de $72,7 \%$, já que três juízes o avaliaram como sendo razoável.

Após validação de conteúdo, a versão final do instrumento para identificação de memórias de pacientes de UTI ficou constituída por nove itens, sendo seu conteúdo semântico agrupado em quatro grandes domínios: memórias relacionadas ao tratamento; memórias do ambiente; memórias relacionadas às experiências emocionais e aquelas relacionadas a memórias irreais ou de ilusão. Para identificação dos tipos de memórias, classificou-se como apresentando amnésia total aqueles pacientes que não tiveram nenhuma recordação do período de internação; memória de fatos reais aqueles com recordações relacionadas a fatos reais referentes ao tratamento, ao ambiente e a eventos emocionais e, por fim, memórias de ilusão relacionadas a eventos irreais, como pesadelos, sonhos e alucinações (Anexo 1). O instrumento validado recebeu a denominação de instrumento de avaliação de memórias de UTI (IAM-UTI).

\section{Aplicabilidade e validade semântica}

Seguindo as recomendações de Pasquali16, após a validação do instrumento, foi realizado um estudo-piloto para verificação da aplicabilidade e validade semântica do instrumento na população-alvo. Para tanto, foi realizado um pré-teste com uma amostra-piloto de pacientes internados na UTI de um hospital universitário.

Foram avaliados 68 pacientes que retornaram ao ambulatório dois meses após a alta da UTI. Destes, mais da metade $(55,9 \%)$ era do sexo masculino. A idade variou entre 18 e 80 anos, com média de 44,8 \pm 18,3 anos. Destes, 11,7\% tinham menos de 20 anos de idade, e a maioria dos pacientes encontrava-se na faixa etária entre 20 e 65 anos (75\%). A maior parte dos pacientes $(41,2 \%)$ foi admitida na UTI para tratamento de disfunções clínicas diversas, 16,2\% se submeteram a procedimento cirúrgico, 25\% foram admitidos em consequência de trauma e 17,6\%, por condições neurológicas. 0 tempo médio de internação variou entre 1 e 41 dias, com uma permanência média de 9,2 \pm 8,3 dias. A distribuição da presença de memórias dos pacientes avaliados segundo sexo, idade, causa de admissão e tempo de permanência na UTI é apresentada na tabela 2.

Do total de pacientes avaliados, 73,5\% apresentaram algum tipo de recordação da sua permanência na UTI, contra 26,5\% que não tinham nenhuma recordação daquele período. Observou-se associação significativa entre as variáveis causa de admissão na UTI e presença de memória $(p=0,019)$. Metade dos pacientes que internaram em razão de trauma não apresentou memórias das experiências vividas na UTI. Por outro lado, pacientes que permaneceram por um período maior do que sete dias internados na UTI também não apresentaram memórias desse período.

Quanto às memórias de fatos reais, $76,5 \%$ recordaram situações relacionadas ao tratamento, $82,3 \%$ tinham recordações acerca do ambiente da UTI e 75\% relataram memórias relacionadas à percepção de seus sentimentos e sensações experimentadas (memórias emocionais). Outros estudos realizados com pacientes de UTI e avaliados com o ICUM, ferramenta que foi base para construção do instrumento ora proposto, também encontraram altas taxas de recordações relatadas pelos pacientes. No estudo realizado por Ringdal et al. ${ }^{2}$ com pacientes vítimas de trauma, $83 \%$ dos pacientes ti-

Tabela 2. Distribuição da presença de memórias dos sujeitos referentes à UTI, segundo características sociodemográficas e clínicas. Cascavel (PR), 2008

\begin{tabular}{|c|c|c|c|c|c|c|}
\hline \multirow{3}{*}{ Variáveis } & \multirow{3}{*}{$(n=68)$} & \multicolumn{4}{|c|}{ Presença de memória } & \multirow{3}{*}{$p$} \\
\hline & & \multicolumn{2}{|c|}{$\operatorname{Sim}(n=50)$} & \multicolumn{2}{|c|}{ Não $(n=18)$} & \\
\hline & & $\mathrm{N}^{0}$ & $\%$ & $\mathrm{~N}^{0}$ & $\%$ & \\
\hline \multicolumn{7}{|l|}{ Sexo } \\
\hline Feminino & 30 & 22 & 44,0 & 8 & 44,4 & 1,0000 \\
\hline Masculino & 38 & 28 & 56,0 & 10 & 55,6 & \\
\hline \multicolumn{7}{|l|}{ Faixa etária } \\
\hline 18-40 anos & 31 & 24 & 48,0 & 7 & 38,9 & \multirow{3}{*}{0,768} \\
\hline $41-65$ & 25 & 18 & 36,0 & 7 & 38,9 & \\
\hline$>65$ anos & 12 & 8 & 16,0 & 4 & 22,2 & \\
\hline \multicolumn{7}{|l|}{ Admissão } \\
\hline Clínica & 28 & 25 & 50,0 & 3 & 16,7 & \multirow{4}{*}{0,019} \\
\hline Cirúrgica & 11 & 8 & 16,0 & 3 & 16,7 & \\
\hline Trauma & 17 & 8 & 16,0 & 9 & 50,0 & \\
\hline Neurológico & 12 & 9 & 18,0 & 3 & 16,7 & \\
\hline \multicolumn{7}{|l|}{ Tempo UTI } \\
\hline Até 48h & 16 & 14 & 28,0 & 2 & 11,1 & \multirow{3}{*}{0,338} \\
\hline 3-7 dias & 21 & 14 & 28,0 & 7 & 38,9 & \\
\hline Mais de 7 dias & 31 & 22 & 44,0 & 9 & 50,0 & \\
\hline
\end{tabular}


nham alguma recordação de fatos reais de sua permanência na UTI, e recordações de memórias emocionais, a exemplo da dor, foram relatadas por $70 \%$ dos pacientes.

Estudo anterior com pacientes avaliados com o ICUM apresentou resultados semelhantes aos do presente estudo. Samuelson et al. ${ }^{10}$ investigaram a relação entre memória e sedação em pacientes submetidos à ventilação mecânica. Dos 250 pacientes avaliados, 82\% relataram memórias da UTI, sendo que $96 \%$ deles tinham memória de fatos reais, com uma média de sete eventos recordados, 88\% apresentaram memórias emocionais e 41\%, memórias de ilusão.

Constatou-se, no presente estudo, uma alta incidência de relatos de memórias ilusórias, e 39,7\% dos pacientes referiram experiências de alucinações, $22 \%$ relataram sonhos, alguns relacionados à vivência de internação, e 14,7\% relataram pesadelos. Os dados de um estudo anterior ${ }^{17}$ mostraram resultados semelhantes a esses. Nesse estudo, os pacientes foram avaliados por um instrumento com questões abertas referentes a memórias de sonhos, pesadelos, alucinações, semelhantes ao item seis do nosso instrumento que avalia o mesmo evento. A incidência de sonhos foi de $21,1 \%$ e, desses, 9,9\% relataram sonhos desagradáveis, sendo classificados pelos pacientes como pesadelos, e 36,6\% relataram alucinações. Já os resultados do estudo de Samuelson et al. ${ }^{10}$ apresentaram altas taxas de memórias de ilusão. Entre os 65 pacientes com memórias de ilusão, $61 \%$ relataram pesadelos, 59\%, alucinações e 16\%, delírios.

\section{DISCUSSÃO}

A partir dos resultados obtidos, o instrumento possibilitou a identificação das recordações das experiências vividas pelos pacientes durante sua permanência em UTI, sendo os itens suficientes para identificar o evento estudado. Além disso, forneceu dados qualitativos acerca das recordações de memórias de ilusão, a exemplo dos sonhos, pesadelos e alucinações, com base no relato desses eventos.

Em relação aos tipos de memórias identificadas pelo instrumento, as mais citadas foram a visita da família, sentir-se confuso e agitado e ter ficado com as mãos e pés amarrados. Já as memórias de ilusão, a exemplo de pesadelos e alucinações, foram descritas como fonte de desconforto e, na maioria das vezes, estavam relacionadas com fragmentos de situações vividas durante a internação na UTI. Esses dados são consistentes com os resultados encontrados na literatura, demonstrando uma variação entre $44 \%$ e $77 \%$ de recordações de memórias de ilusão' ${ }^{12}$.

Alguns itens sofreram alterações e grande parte das sugestões dos peritos foi acatada. Por exemplo, foram propostas e acatadas as mudanças no item oito quanto à apresentação do enunciado, sendo observada maior clareza após sua mudança. O referido item inicialmente foi apresentado da seguinte forma: "Você sabia que lugar era aquele durante o período em que estava na UTI? Com as sugestões dos juízes, a nova construção ficou assim enunciada: "No período de internação na UTI, houve momentos em que você não sabia onde estava? Como foram realizadas poucas alterações, não houve necessidade de submeter novamente o instrumento reformulado a um novo parecer dos experts. Na análise do quesito precisão, o item não deveria possuir elementos supérfluos, deveria ter uma posição definida no contínuo do atributo e ser diferente das questões que englobavam o mesmo contínuo ${ }^{16}$. Um item que não é simples, que não expressa ideia única, traz confusão ao respondente ${ }^{16}$, assim, apesar de a maioria dos itens ter apresentado validade de conteúdo, alguns enunciados passaram por alguns ajustes a partir das sugestões dos experts e, depois de modificados, optou-se por não descartar nenhum item.

A opção em manter os itens seis e oito, os mais discordantes entre os juízes, se deve ao elevado número de pacientes que relatam suas vivências de sonhos, pesadelos, confusão mental e alucinações durante a permanência em UTI, constatado tanto nos resultados de estudos anteriores ${ }^{9,12}$ quanto na prática clínica. Além disso, no quesito relevância e precisão, este item obteve uma porcentagem acima de $80 \%$ de concordância, estando em conformidade com o critério de decisão adotado no estudo para determinar a qualidade do item.

A partir do teste-piloto, todos os itens se mostraram compreensíveis aos pacientes. Contudo, nos itens com questões abertas, observou-se que a maioria dos pacientes apresentou dificuldade em respondê-los. Mas, após iniciar os itens em forma de cheklist, os pacientes foram indicando suas recordações daquele período sem dificuldades. Dessa forma, observou-se que, para avaliar as recordações de pacientes após a alta da UTI, instrumentos tipo cheklist parecem ser mais adequados do que aqueles com questões abertas.

O instrumento obteve uma avaliação global positiva em relação aos sete quesitos propostos: objetividade, simplicidade, relevância, precisão, credibilidade, clareza e adequação da linguagem, indicando que os itens apresentados são adequados e pertinentes para avaliação de aspectos relacionados ao ambiente de terapia intensiva, além de se mostrarem compreensíveis pela população avaliada, sugerindo-se que o instrumento apresenta validade de conteúdo. Portanto, pode-se afirmar que o instrumento proposto foi considerado válido no que se refere ao conteúdo e à aplicabilidade na população de pacientes internados em UTI.

Algumas limitações devem ser destacadas nesse estudo. Primeiro, foi realizada somente a validação de conteúdo do instrumento, fazendo-se necessário, para uso em pesquisas, conhecer suas propriedades psicométricas a partir das demais etapas de validação. Segundo, sendo a maioria dos itens do tipo checkllist, não permitiu ao paciente resgatar da memória sua experiência, incorrendo o erro de induzir sua resposta, embora a opção por essa forma de apresentação deva-se à espe- 
cificidade dos pacientes avaliados. Pacientes de UTI geralmente estão gravemente enfermos, permanecem sedados, são admitidos, na maioria das vezes, devido a traumatismo cranioencefálico e sua capacidade de evocar recordações pode está diminuída em decorrência das várias alterações cognitivas (atenção, condições emocionais, percepção, motivação etc.) apresentadas pelo paciente durante a internação, podendo, dessa forma, dificultar o resgate de suas lembranças.

Além disso, poderia ocorrer dificuldade na formação de traços mnêmicos, na consolidação e na sua recuperação, como foi observado durante aplicação dos itens 2 e 9 do instrumento, em que se solicita ao paciente que descreva suas recordações e experiências desagradáveis vividas na UTI, observando-se que a maioria dos pacientes não conseguia relatar suas lembranças. Há que se considerar, também, que possibilitar livremente o resgate das experiências do paciente poderia ocorrer que somente parte da memória seria restituída ou, ainda, pensamentos e associações poderiam ser confundidos devido às condições clínicas do paciente.

Dessa forma, muitas recordações acerca dos diversos aspectos relacionados ao tratamento, ao ambiente e aos aspectos emocionais poderiam não ser evocadas e exploradas, como ocorreu pela forma de apresentação do instrumento proposto. É frequente que, quando se evoca uma dada memória, somente parte dela seja restituída. De fato, a memória é extremamente dinâmica e lembrar implica um processo ativo de reconstrução. E, por fim, as lembranças do paciente podem ter a interferência das conversas tidas com os familiares sobre o período de internação na UTI e sua recordação pode estar relacionada às informações repassadas pelos familiares e não propriamente sua vivência.

A falta de instrumentos para avaliar as experiências vividas pelo paciente durante a internação em UTI aponta para a necessidade da construção e validação de instrumentos para identificação desse evento. Em função da carência desse tipo de ferramenta para essa população específica, evidencia-se a relevância de dar continuidade à adequação do referido instrumento. Recomenda-se sua aplicação em outros contextos para confirmação dos resultados e testar a consistência dos itens. Dessa forma, outros estudos devem ser empreendidos para comprovação das demais etapas que envolvem a validação do instrumento ora apresentado.

\section{CONCLUSÃO}

Com base no julgamento dos experts, constatou-se que a maioria dos itens foi avaliada de forma positiva, alcançando uma concordância, no geral, entre 90,9\% e 100\%. Desse modo, os itens propostos mostraram-se compreensíveis, claros e com uma adequada estrutura de apresentação. O instrumento apresenta validade de conteúdo e semântica atestada por um painel de experts e pela população-alvo, demonstran- do possuir características teóricas para identificar memórias de pacientes internados em UTI a curto e longo prazo.

\section{AGRADECIMENTO}

À professora Dra. Rosangela Getirana Santana, do Departamento de Estatística da Universidade Estadual de Maringá (UEM), pelo apoio na construção do instrumento e pela revisão e auxílio na análise estatística.

Conflito de interesse: os autores declaram não existir conflito de interesse.

\section{REFERÊNCIAS}

1. Capuzzo M, Pinamonti A, Cingolani E, Grassi L, Bianconi M, Contu P, et al. Analgesia, sedation, and memory of intensive care. J Crit Care. 2001;16:83-9.

2. Ringdal M, Johansson L, Lundberg D, Bergbom I. Delusional memories from the intensive care unit - Experienced by patients with physical trauma. Intensive Crit Care Nurs. 2006;22:346-54.

3. Samuelson KAM, Lunderberg D, Fridlund B. Stressful memories and psychological distress in adult mechanically ventilated intensive care patients - A 2 month follow-up study. Acta Anaesthesiol Scand. 2007;51:671-8.

4. Granberg A, Bergbom-Engberg I, Lundberg D. Acute confusion and unreal experiences in intensive care patients in relation to ICU syndrome Part II. Intensive Crit Care Nurs. 1999;15:19-33

5. Roberts B, Chaboyer W. Patients' dreams and unreal experiences following intensive care unit admission. Nurs Crit Care. 2004;9(4):173-80.

6. Hupcey JE. Feeling safe: the psychosocial needs of ICU patients. J Nurs Scholarship. 2000;32(4):361-7.

7. Rattray J, Johnston M, Wildsmith JAW. The intensive care experience: development of the Intensive Care Experience (ICE) Questionnaire. J Adv Nurs. 2004;47:64-73.

8. Roberts BL, Rickard CM, Rajbhandaric D, Reynolds P. Patients' dreams in ICU: Recall at two years post discharge and comparison to delirium status during ICU admission: a multicentre cohort study. Intensive Crit Care Nurs. 2006;22:264-73.

9. Van de Luer PJ, Schans CP, Loef BG, Deelman BG, Geertzen JHB, Zwaveling JH. Discomfort and factual recollection in intensive care unit patients. Crit Care Med. 2004;8(6):67-72.

10. Samuelson KAM, Lunderberg D, Fridlund B. Memory in relation to depth of sedation in adult mechanically ventilated intensive care patients. Intensive Care Med. 2006;32:660-7.

11. Adamson H, Murgo M, Boyle M, Kerr S, Crawford M, Elliott D. Memories of intensive care and experiences of survivors of a critical illness: an interview study. Intensive Crit Care Nurs. 2004;20:257-63.

12. Jones C, Griffiths RD, Humphris G, Skirrow PM. Memory, delusions, and the development of acute posttraumatic stress disorder-related symptoms after intensive care. Crit Care Med. 2001;29:573-80.

13. Capuzzo M, Valpondi V, Cingolani E, De Luca S, Gianstefani G, Grassi L, et al. Application of the Italian version of the Intensive Care Unit Memory tool in the clinical setting. Crit Care Med. 2004;8(1):48-55.

14. Jones $C$, Griffiths RD, Humphris $G$. Disturbed memory and amnesia related to intensive care. Memory. 2000;8:79-94.

15 Jones C, Humphris G, Griffiths RD. Preliminary validation of the ICUM tool: a tool for assessing memory of the intensive care experience. Clin Intensive Care. 2000;11:251-5.

16. Pasquali L. Princípios de elaboração de escalas psicológicas. Rev Psiq Clín. 1998;25(5):20613. Disponível em: http:www.henet.usp.br/ipq/revista/r255/conc255a.htm. Acesso em: agosto de 2008.

17. Rundshagen I, Schnabel K, Wegner C, Schulte J. Incidence of recall, nightmares, and hallucinations during analog sedation in intensive care. Intensive Care Med. 2002;28:38-43. 
Anexo 1. Instrumento para avaliação de memórias (IAM-UTI)

Nome:

Tempo da alta da UTI:

1. Você se recorda do período que ficou internado na UTI?（） Sim ( ) Não

2. Do que você se recorda?

3. Você se recorda (aspectos relacionados ao tratamento/à memória de fatos reais):
$\square$ Da admissão/entrada na UTI
$\square$ Da sonda no nariz
$\square$ Dos aparelhos ao seu redor
$\square$ Da máscara de oxigênio
$\square$ Do tubo endotraqueal na boca
$\square$ Da sonda vesical (para fazer xixi)
$\square$ Dos procedimentos realizados/exames/radiografia
$\square$ Da aspiração (caninho para retirar a secreção)
$\square$ Da extubação (retirada do tubo)
$\square$ Higiene/banho/escovação

4. Você se recorda (aspectos relacionados ao ambiente/à memória de fatos reais):
$\square$ Do barulho dos aparelhos
$\square$ Dos outros pacientes
$\square$ Da visita dos familiares
$\square$ Das informações dadas pela equipe (enfermagem, médico)
$\square$ Das conversas da equipe
$\square$ Das luzes
$\square$ Da alta da UTI para enfermaria geral

5. Você se recorda de (aspectos relacionados a memórias de experiências emocionais e físicas):
$\square$ Ter sentido dor
$\square$ Sentir-se sozinho
$\square$ Ter ficado agitado
$\square$ Sentir-se desanimado
$\square$ Sentir-se com medo, ansioso
$\square$ Sentirfome/sede
$\square$ Ter ficado confuso
$\square$ Sentir dificuldade de dormir
$\square$ Ter ficado desorientado (tempo/espaço)
$\square$ Dificuldade de respirar
$\square$ Ter ficado contido/com as mãos amarradas
$\square$ Dificuldade para falar/se comunicar

6. Você se recorda de (aspectos relacionados a memórias ilusórias):
$\square$ Sonhos
$\square$ Pesadelos
$\square$ Alucinações

Foi uma experiência:
$\square$ Ruim
$\square$ Boa
$\square$ Indiferente

7. Após a alta hospitalar, você teve sonhos, pesadelos referentes ao período da UTI? （） Sim ( ) Não Data da entrevista:

$$
\text { I__ } 1
$$

8. No período de internação na UTI, houve momentos em que você não sabia onde estava? （） Sim（） Não

9. Qual foi a experiência mais desagradável durante a sua internação na UTI?

Resultado:

$\square$ Amnésia total

Memória de fatos reais

Memórias ilusórias

Data da avaliação: I 